DOI: https://doi.org/10.11144/Javeriana.upsy17-5.tcmj

\title{
Transformar conflictos motores mediante los juegos cooperativos en Educación Primaria*
}

\section{Transforming Motor Conflicts through Cooperative Games in Primary School}

Recepción: 19 Julio 2016 | Aceptación: 06 Junio 2018

\author{
Unai Sáez de OcÁriz Granja ${ }^{a}$ \\ Instituto Nacional de Educación Física de Cataluña, \\ España \\ ORCID: http://orcid.org/0000-0003-1083-2575 \\ Pere Lavega-Burgues \\ Instituto Nacional de Educación Física de Cataluña, \\ España \\ ORCID: http://orcid.org/0000-0001-9622-054X \\ JAUME MARCH-JanÉs \\ Universitat de Lleida, España \\ ORCID: http://orcid.org/0000-0002-5977-8744 \\ Jorge Serna Bardavío \\ Instituto Nacional de Educación Física de Cataluña, \\ España \\ ORCID: http://orcid.org/0000-0002-5454-9790
}

a Autor de Correspondencia. Correo electrónico: usaez@gencat.cat

Para citar este artículo: Sáez de Ocáriz Granja, U., Lavega-Burgues, P., March-Janés, J., \& Serna Bardavío, J. (2018). Transformar conflictos motores mediante los juegos cooperativos en Educación Primaria. Universitas Psychologica, 17(5), 1-13. http:// doi.org/10.11144/Javeriana.upsy17-5.tcmj

\section{RESUMEN}

Esta investigación examinó el tipo de conflictos motores (CM) originados por un programa de intervención educativa basado en juegos cooperativos desarrollados en las clases de Educación Física, en un centro de educación primaria, con el propósito de identificar el índice de intensidad de los $\mathrm{CM}$, como instrumento docente para la optimización de la convivencia escolar. Participaron 43 alumnos, 22 niñas y 21 niños, a lo largo de un curso escolar. Se consideraron 42 clases de Educación Física, donde se plantearon 117 juegos de cooperación y se registraron 319 CM. Entre las conclusiones más notables se puede subrayar la aplicabilidad del índice de conflictividad como herramienta en la educación de los conflictos para fomentar el desarrollo de la convivencia en las escuelas.

Palabras clave

relación entre estudiantes; educación física; índice de conflictividad; cooperación; teoría acción motriz.

\begin{abstract}
This research examined the type of motor conflicts (MC) that led to an educational intervention program based in developed cooperative games in physical education classes in a primary education school and identify the intensity index of motor conflicts as a teaching instrument for improvement of school coexistence. 43 primary school students, 22 girls and 21 boys participated throughout the school year. 42 physical education classes were considered in which 117 cooperative games and $319 \mathrm{MC}$ were registered. Among the most important findings we can highlight the applicability of conflict index as a tool for learning how to live together and to encourage the development of coexistence in schools.
\end{abstract}


Keywords

student school relationship; physical education; conflict index; cooperation; motor action domain.

\section{Introducción}

En una sociedad en continuo cambio como la actual, la vida en comunidad no solo significa compartir un mismo espacio, sino que lleva implícita la interacción permanente entre diferentes personas con el objetivo de desarrollar actuaciones proactivas y constructivas a través de aprendizajes democráticos (Badia, 2009). En las sociedades plurales, fundamentadas en la cordialidad y en el respeto, la convivencia es una de las prioridades educativas clave para el desarrollo de la identidad personal y grupal de sus miembros (Led \& Quera, 2007).

La vida en sociedad lleva implícita la participación en numerosas experiencias de relación interpersonal. En este diálogo social, resulta inevitable la aparición de conflictos originados por intereses opuestos en las relaciones sociales que pueden originar tensiones (Molina, 2005; Ortega \& Del Rey, 2003; Sáez de Ocáriz \& Lavega, 2013). En este sentido, la convivencia y el conflicto, realidades paralelas e inherentes al ser humano, son un potente instrumento de transformación social (Ceballos, Rodríguez, Correa, \& Rodríguez, 2016; Viguer \& Solé, 2015). Dependiendo de cómo estas se gestionen, pueden llegar a ser una magnífica herramienta que permita la evolución constructiva de sus protagonistas (Jares, 2006; Pérez-Gallardo, Álvarez-Hernández, Aguilar, Fernández-Campoy, \& Salguero, 2013; Sáez de Ocáriz, 2011).

\section{Escuela, conflicto y convivencia}

Los conflictos interpersonales siguen teniendo una gran presencia en las escuelas (ÁlvarezGarcía, Rodríguez, González-Castro, Nun\#ez, \& Álvarez, 2010; Ceballos et al., 2012; Garaigordobil \& Oñederra, 2009; Gázquez, Pérez-Fuentes, Carrión, \& Santiuste, 2010; Sáez de Ocáriz, 2011; Torrego \& Galán, 2008). Sin embargo, más que considerarse únicamente como una situación negativa que se debe evitar, también son una buena oportunidad para la construcción de relaciones interpersonales positivas que permitan educar la convivencia en el alumnado (Ararteko, 2006; Jares, 2006; Martín, Fernández, Andrés, Del Barrio, \& Echeita, 2003; Penalva, Hernández, \& Guerrero, 2014). La escuela proporciona un contexto social de primer orden para educar dicha convivencia democrática que puede favorecer un adecuado desarrollo intelectual, social y moral de los futuros ciudadanos (Álvarez-García et al., 2010; Led \& Quera, 2007; Pérez-Pérez, 2007; Torregrosa, Inglés, García-Fernández, Valle, \& Núñez, 2012).

Dichas afirmaciones ponen de manifiesto la necesidad de una formación integral, tanto del alumnado como del profesorado, fundamentada en el pensamiento crítico, la participación democrática en equidad y la gestión positiva del conflicto y las emociones (Ascorra, Bilbao, Gómez, \& Morales, 2016; Badia, 2009; GilMadrona \& Martínez-López, 2015; Torrecilla, Olmos, \& Rodríguez, 2016). En consecuencia, la Unión Europea establece como política prioritaria la educación de la convivencia escolar (Molina, 2005; Ortega \& Del Rey, 2003; Sáez de Ocáriz, Lavega, \& March, 2013). En España, el Ministerio de Educación y Ciencia también determina como objetivo prioritario en la Educación Primaria (Real Decreto. Ministerio de Educación y Ciencia, 2006), el desarrollo de la competencia social y ciudadana. El Ministerio español indica que esas competencias deberían basarse en el respeto y el aprendizaje cooperativo para enseñar al alumnado a afrontar la convivencia y los conflictos con una actitud constructiva, solidaria y responsable (Badia, 2009; Garaigordobil, 2012; Led \& Quera, 2007).

Paralelamente, diversos estudios han confirmado que el desarrollo de las habilidades prosociales es una competencia imprescindible para la transformación constructiva de las situaciones conflictivas (Hromek \& Roffey, 2009; Johnson \& Johnson, 1999; Lederach, 1995; León, Gozalo, \& Polo, 2012). También se ha constatado que los alumnos socialmente competentes muestran niveles adecuados de 
razonamiento moral, así como de inteligencia intrapersonal e interpersonal, necesarias para una correcta adaptación a la realidad tanto social como laboral (Durán Aponte \& Durán García, 2012; Led \& Quera, 2007).

\section{El conflicto desde la perspectiva de género}

Las investigaciones sobre la convivencia escolar constatan que los chicos no se comportan igual que las chicas cuando protagonizan situaciones conflictivas (Díaz-Aguado, 2005; Inglés et al., 2008; Sáez de Ocáriz, 2011; Toldos, 2005). Dicha dualidad tiende a asociar lo masculino con la agresividad, la competitividad, la insensibilidad y lo femenino, con la empatía, la sensibilidad social y la comprensión; perspectiva que no hace más que contribuir a la reproducción de un modelo de relación social basado en estereotipos culturales, como pueden ser los casos del sexismo y el machismo (Díaz-Aguado, 2005; Díaz-Aguado \& Martín-Seoane, 2011).

Las evidencias científicas muestran que el género masculino suele protagonizar mayor número de conflictos y de mayor intensidad que el femenino; los chicos acostumbran a protagonizar más conductas agresivas vinculadas a agresiones físicas, mientras que en las chicas son más habituales las agresiones verbales e indirectas (Díaz-Aguado, 2005; Inglés et al., 2008; Österman et al., 1998; Toldos, 2005). Asimismo, se complementa esta visión afirmando que las chicas presentan niveles más altos de conductas prosociales que los chicos (Calvo, González, \& Martorell, 2001).

Sin embargo, es necesario considerar que dichos resultados no solo están ligados al sexo, cuestiones biológicas, sino que se encuentran estrechamente conectados a la generalización transcultural de los estereotipos de género reinantes en el contexto cultural en el que participan los protagonistas (Díaz-Aguado, 2005; Díaz-Aguado \& Martín-Seoane, 2011; Toldos, 2005). De ahí que el uso los juegos, en tanto que manifestaciones sociales, puedan ser de gran interés para la educación de las relaciones interpersonales (Parlebas, 2001).

Por otro lado, se ha visto que el papel del profesorado ante los conflictos escolares y la mejora de la convivencia escolar resulta fundamental (Álvarez-García et al., 2010; DíazAguado \& Martín-Seoane, 2011; Penalva et al., 2014). Sin embargo, tal y como confirman diferentes estudios, se observa que la formación que reciben los docentes en este ámbito es escasa, de ahí la conveniencia de generar investigaciones como la del presente trabajo (Álvarez-García et al., 2010; Jares, 2006; Martín et al., 2003).

\section{Educar las relaciones interpersonales desde la educación física}

La educación física interviene a través de aprendizajes procedimentales, es decir, las enseñanzas son fruto de la adquisición y transformación de conductas motrices (Parlebas, 2001). Cada juego motor dispone de una lógica u orden interno que exige a cualquier participante relacionarse con otras personas, con el espacio, con el tiempo y con los objetos (Lagardera \& Lavega, 2005). Fruto de esas relaciones, cada alumno adapta sus respuestas a esa lógica interna del juego a través de diferentes conductas motrices.

El concepto sistémico de conducta motriz establece que cualquier respuesta motriz ( $\mathrm{p}$. ej., un pase a un compañero) activa de manera unitaria toda su personalidad, de manera que ese pase también pone en acción las dimensiones orgánica (implicación fisiológica), afectiva (reacciones emocionales), cognitiva (toma de decisiones) y social (tipo de relaciones con los compañeros de juego) (Parlebas, 2001). Así pues, entender la educación física como la pedagogía de las conductas motrices, permite al docente aportar experiencias significativas a la formación integral del alumnado (Tabla 1) (Lagardera \& Lavega, 2005; Lavega, Planas, \& Ruíz, 2014; Sáez de Ocáriz et al., 2013).

Educar conductas motrices implica formar la convivencia escolar a través de la trasformación de las intervenciones del alumnado cuando 
protagonizan conflictos interpersonales en las sesiones de educación física (Sáez de Ocáriz et al., 2013). Es por ello que el juego motor ejerce un papel clave en la educación de los conflictos interpersonales, ya que puede generar en el alumnado experiencias agradables en las que es más fácil favorecer aprendizajes prosociales (Hromek \& Roffey, 2009; Johnson \& Johnson, 1999; Parlebas, 2001).

La teoría de la acción motriz confirma la extraordinaria diversidad de relaciones que originan los juegos motores. Así, atendiendo al tipo de interacción motriz, se identifican cuatro grandes familias de experiencias (o dominios de acción motriz) (Parlebas, 2001): a) juegos psicomotores donde no existe interacción motriz entre los participantes y juegos sociomotores donde la interacción motriz existente puede ser de colaboración, b) de cooperación (cuando dos o más participantes se ayudan mutuamente para alcanzar un objetivo común), c) de oposición (dos o más protagonistas rivalizan por el objetivo del juego) y d) de cooperación-oposición (varios jugadores cooperan entre ellos y se oponen a un equipo de adversarios).

\section{El conflicto motor en las situaciones motrices de cooperación}

De las cuatro clases de experiencias motrices merecen especial atención los juegos cooperativos. Se trata de una familia de juegos a menudo poco aplicada en los programas de educación física (Johnson \& Johnson, 1999; Lavega et al., 2014). Sin embargo, son prácticas muy adecuadas para favorecer el desarrollo de competencias sociales y la creación de ambientes positivos en las clases de educación física (Koçak, 2008; León et al., 2012; Pérez \& Poveda, 2008).

Cada juego motor cooperativo se comporta como un auténtico laboratorio de relaciones sociales (Parlebas, 2001). Cualquier alumno al cooperar se pone a prueba en el desafío de compartir relaciones positivas con otras personas, tanto en el momento de pactar la estrategia que se va a seguir como en el momento de ejecutar las respuestas acordadas. Cuando las conductas motrices son cordiales (ajustadas), la convivencia se desarrolla en un clima de relaciones favorables. En cambio, en ocasiones, puede ocurrir que aparezcan desacuerdos, tensiones y desequilibrios en la relación que se manifiestan mediante conductas motrices que no favorecen dicha relación interpersonal. Así, un conflicto motor puede originarse a través de una conducta verbal de pacto desajustada (en el momento de establecer el acuerdo con los otros participantes), una conducta motriz desajustada (que supone desviarse de las respuestas esperadas que favorecen el diálogo motriz entre los participantes) o una conducta motriz perversa (que genera desorden y que implica infringir las reglas del juego). En consecuencia, las personas afectadas pueden reaccionar mediante una agresión verbal (p. ej., insulto), física (p. ej., empujón) o mixta (p. ej., insulto más empujón) (Sáez de Ocáriz, 2011).

Tabla 1

Tipos de conductas sociales en el juego de los pases

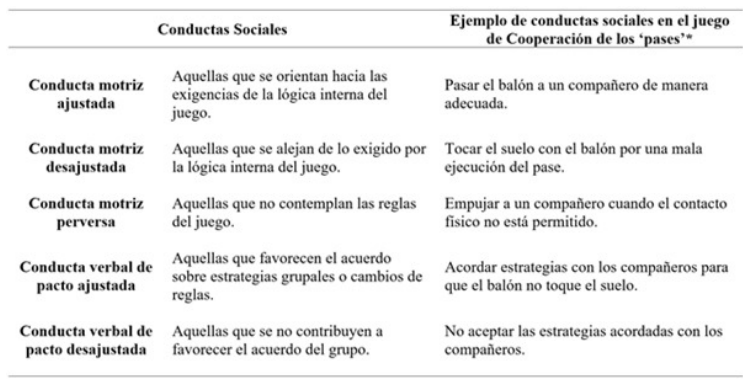

* Se solicita a los miembros que realicen el máximo de pases seguidos sin que el balón toque el suelo.

Atendiendo a la perspectiva de género, los estudios científicos sobre los juegos tradicionales en España (Etxebeste, 2012; Lavega, 2009; Lavega et al., 2014) indican que los juegos cooperativos están mucho más presentes en las chicas que en los chicos. En estos juegos la ayuda mutua, el sacrificio por los demás, la lentitud de las acciones y la capacidad de interactuar muy propias del género femenino son factores clave para obtener el éxito colectivo. Sin embargo, la fuerza, la agresividad o el esfuerzo físico mucho más presentes en el género masculino no tienen un papel determinante. Por ello, dada la naturaleza social y cultural del conflicto, se puede 
esperar que cuando ambos géneros participan en este tipo de juegos, sus conflictos sean desiguales.

\section{El índice de conflictividad de los conflictos motores de cooperación}

Una vez detectados dichos conflictos, el profesor de educación física necesita de herramientas y recursos para transformar educativamente las situaciones de desequilibrio generadas, buscando la emergencia de experiencias positivas que permitan el desarrollo integral de sus estudiantes (Sáez de Ocáriz, 2011). Para ello, un elemento de gran interés e importancia para la educación de los conflictos es el índice de conflictividad (ICF), que permite cuantificar el nivel conflictivo del alumnado para, en consecuencia, poder plantear, diseñar y poner en práctica estrategias coherentes para la optimización de sus conductas conflictivas (Sáez de Ocáriz \& Lavega, 2013).

El ICF es el resultante de la intensidad del origen, donde se consideran tres opciones de conductas conflictivas (de pacto $=1$; desajustada $=2$; perversa $=3$ ), y la intensidad de la reacción que caracteriza al conflicto motor, en la que se encuentran tres tipos de respuestas conflictivas (agresión verbal $=1$; agresión física $=2$; agresión mixta, es decir, verbal y física simultáneas $=3$ ). En ambos casos, las puntuaciones van del 1 al 3 en función de su nivel de intensidad, siendo el 1 la menos intensa y el 3 la de mayor intensidad (Tabla 2).

\section{Tabla 2}

Clasificación de los conflictos motores

\begin{tabular}{|c|c|c|c|c|c|c|}
\hline $\begin{array}{r}\text { In } \\
\text { conf }\end{array}$ & $\begin{array}{l}\text { ice de } \\
\text { ctividad }\end{array}$ & Origen & Int & Respuesta & Int & $\begin{array}{c}\text { Int } \\
\text { Total }\end{array}$ \\
\hline \multirow{3}{*}{ Bajo } & Tipo I & Conducta Verbal de Pacto Conflictiva & 1 & Agresión Verbal & 1 & 2 \\
\hline & Tipo II & Conducta Verbal de Pacto Conflictiva & i & Agresión Fisica & 2 & 3 \\
\hline & Tipo III & $\begin{array}{l}\text { Conducta Motriz Desajustada } \\
\text { Conflictiva }\end{array}$ & 2 & Agresión Verbal & 1 & 3 \\
\hline \multirow{3}{*}{ Medio } & Tipo IV & Conducta Verbal de Pacto Conflictiva & 1 & Agresión Mixta & 3 & 4 \\
\hline & Tipo V & Conducta Motriz Desajustada & 2 & Agresión Física & 2 & 4 \\
\hline & Tipo VI & Conducta Motriz Perversa Conflictiva & 3 & Agresión Verbal & 1 & 4 \\
\hline \multirow{3}{*}{ Alto } & Tipo VII & Conducta Motriz Desajustada & 2 & Agresión Mixta & 3 & 5 \\
\hline & Tipo VIII & Conducta Motriz Perversa Conflictiva & 3 & Agresión Fisica & 2 & 5 \\
\hline & Tipo IX & Conducta Motriz Perversa Conflictiva & 3 & Agresión Mixta & 3 & 6 \\
\hline
\end{tabular}

Nota. Int: intensidad.

Fuente: elaboración propia (adaptado de Sáez de Ocáriz \& Lavega, 2013, 2015; Sáez de Ocáriz, 2011).

Para educar en el conflicto, una vez que se conoce el ICF o perfil conflictivo del grupo, el profesor puede introducir medidas de prevención y/o intervención directa con el objetivo de modificar los aprendizajes y favorecer la comunicación y el dialogo interpersonal de sus alumnos (Lederach, 1995; Sáez de Ocáriz, 2011). Estas estrategias inciden principalmente sobre: a) la lógica interna del juego, donde es posible modificar las reglas de la práctica introduciendo normas más favorables o incluso, si dicha modificación no funciona, cambiar de juego buscando condiciones de la lógica interna más propicias y b) los protagonistas del conflicto, donde el docente, dependiendo del índice de conflictividad, puede optar por intervenir directamente o proporcionar al alumnado implicado de un espacio y un tiempo para que estos gestionen el conflicto (Johnson \& Johnson, 1999; Sáez de Ocáriz, 2011).

Atendiendo a la predisposición desigual de chicos y chicas para participar en los juegos cooperativos (Etxebeste, 2012; Lavega, 2009; Lavega et al., 2014), es probable que el índice de conflictividad sea distinto entre ambos géneros, circunstancia que podría originar la combinación de diferentes estrategias, a partir de los criterios mencionados anteriormente, para transformar los conflictos motores.

Dada la escasez de contribuciones científicas en torno al estudio de los conflictos en las clases de educación física (Ararteko, 2006), esta investigación se planteó como objetivo principal examinar el tipo de conflictos motores que originó en chicos y chicas la intervención en un programa basado en juegos cooperativos, así como la aplicabilidad del índice de conflictividad como recurso docente para la mejora de la convivencia escolar.

\section{Método}

El diseño de la investigación correspondió a una observación participante, donde se pudo confirmar, cambiar, modificar o ampliar el conocimiento sobre el objeto de estudio (Quivy \& Campenhoudt, 2000). Asimismo, la información registrada de cada protagonista que participa en un proceso educativo único, 
irrepetible y singular, permitió al investigador observar y analizar el fenómeno, hasta ese momento inasequible (Rodríguez, Gil, \& García, 1996).

\section{Contextualización de la experiencia pedagógica}

La experiencia pedagógica estudiada formaba parte de un programa de educación física específico solicitado por el centro educativo a través de un plan de autonomía de centro para reducir la violencia y el absentismo escolar. Los participantes eran niños y niñas de un nivel socioeconómico y cultural muy bajo, donde la desestructuración familiar era un rasgo común entre todos ellos. Dicha experiencia se realizó durante un curso escolar, a través de la propuesta de juegos sociomotores de cooperación, en 42 sesiones de una hora de duración, dos veces por semana.

\section{Participantes}

El estudio se llevó a cabo con 43 alumnos de Educación Primaria, 21 niños y 22 niñas, de un centro educativo de la ciudad de Lleida (España) de edades comprendidas entre los $8 \mathrm{y}$ los 11 años $(\mathrm{M}=9.35 ; \mathrm{DE}=1.07)$. Del total de participantes, 23 de ellos estudiaban ciclo medio ( 11 chicos y 12 chicas; 8-9 años) y los 20 restantes, ciclo superior (10 chicos y 10 chicas; 10-11 años). En todo momento, se respetaron los principios éticos de las investigaciones sociales (Howe \& Moses, 1999; Mesía, 2007), donde los sujetos no corrieron riesgos, no se produjeron interferencias entre objetivos, se garantizó la privacidad y el respeto a la dignidad personal y el trato al alumnado fue justo.

\section{Procedimiento}

Con el ánimo de optimizar el proceso de enseñanza-aprendizaje, en este estudio, el profesor de educación física asumió el rol de maestro e investigador de forma simultánea (Stenhouse, 1987). Diseñó y puso en práctica 117 juegos de cooperación distribuidos en diferentes sesiones. Al acabar cada una de las sesiones, el profesor/investigador describió cada una de las situaciones motrices propuestas y los procesos de conflicto motor, detectados de una manera concisa y detallada. Se utilizó la observación participante como procedimiento de observación de lo ocurrido en las sesiones de educación física para captar la propia realidad y, posteriormente, reconstruir y describir la globalidad del fenómeno (Quivy \& Campenhoudt, 2000; RuizOlabuénaga, 1999). Esto permitió identificar con rigor metodológico la información obtenida, de la cual se generó una gran base de datos, identificando la información sobre cada una de las variables y categorías, que posteriormente se pasó al formato de SPSS para poder analizarlas estadísticamente.

Dadas las características de los participantes y el centro educativo en el que se realizó la experiencia, se desestimó el uso de recursos audiovisuales y se llevó a cabo un registro narrativo mediante notas de campo al acabar cada clase (véase apartado Instrumentos). Se consideró el registro y análisis de unidades de información molares, cuyo requerimiento era el tener calidad de información referente al tipo de situación motriz realizada y cada uno de los conflictos motores que protagonizaba el alumno al jugar; para ello, se calculó la fiabilidad entre observadores mediante la prueba de Kappa.

\section{Instrumentos}

Para registrar toda la información y posteriormente organizarla, se optó por la utilización de notas de campo como sistema narrativo (Del Rincón, Arnal, Latorre, \& Sans, 1995). Al finalizar cada una de las sesiones de educación física, se registraron dichas notas correspondientes a variables relativas al juego y al conflicto motor, con el objetivo de describir de forma exhaustiva los procesos que ocurrieron durante esa experiencia pedagógica. De esta manera, se evitaron los problemas de registro ocasionados por la observación de fenómenos de tipo social (Del Rincón et al., 1995). 
El índice de conflictividad se elaboró a partir del marco teórico de referencia. La validez de contenido se estableció por medio de la valoración de doce jueces expertos en juegos deportivos y praxiología motriz a partir del marco teórico definido en Lavega (2009). La validez de criterio se determinó a través de la comparación de resultados de los observadores con respecto a los realizados por un experto (índices de Kappa: 56, observadores $\mathrm{K}=0.8-0.86$ y 11 observadores $\mathrm{K}=0.77$ ) (Sáez de Ocáriz \& Lavega, 2013).

\section{Análisis de los datos}

Las variables categóricas se analizaron mediante la prueba estadística chi-cuadrado de Pearson. A posteriori, en aquellas relaciones estadísticamente significativas, se estudiaron los residuos corregidos con el objetivo de profundizar en las relaciones entre variables. Las variables estudiadas fueron: el índice de conflictividad, el agente generador de los conflictos motores, la respuesta conflictiva y el género de los protagonistas. Se utilizó el paquete estadístico Statistical Package for the Social Sciencies (SPSS) versión 19.0 para Windows.

\section{Resultados}

El conflicto motor y el comportamiento del género de sus protagonistas en las situaciones motrices de cooperación

En los 117 juegos sociomotores de cooperación desarrollados durante la experiencia, se detectó algún tipo de conflicto motor (CM) en 64 de ellos (54.7 \%), de un total de 319. Los datos muestran como estos fueron originados por conductas verbales de pacto en un $15.7 \%(n=50)$, por conductas motrices desajustadas en un $33.5 \%$ ( $=107)$ y en un $50.8 \%(n=162)$ por conductas motrices perversas; mientras que las respuestas conflictivas derivadas estuvieron formadas por agresiones verbales en un $53.3 \%(n=170)$, por agresiones físicas en un $16 \%(n=51)$ y en un $30.7 \%(n=98)$ por agresiones mixtas (Figura 1$)$.
Figura 1

El origen y la reacción de los conflictos cooperativos

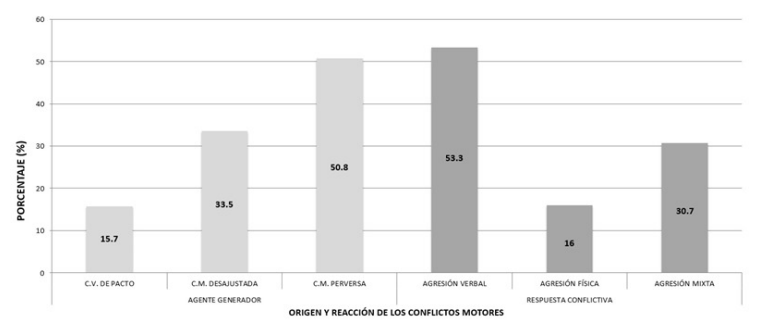

Desde la perspectiva de género, se observó que de los 319 conflictos motores, un $48.3 \%$ fueron conflictos entre chicos $(n=154)$, un $34.5 \%$ entre chicos y chicas $(n=110)$ y un $17.2 \%$ entre chicas $(n=55)$. El origen de estos conflictos fue protagonizado por chicos en un $69 \%(n=220)$ y por chicas en un $31 \%(n=99)$. La reacción al conflicto se presentó en un $62.1 \%$ en los chicos $(n=198)$ y en un $37.9 \%$ en las chicas $(n=121)$ (Figura 2).

\section{Figura 2}

Participación de las chicas y los chicos en el origen y la reacción de los conflictos cooperativos

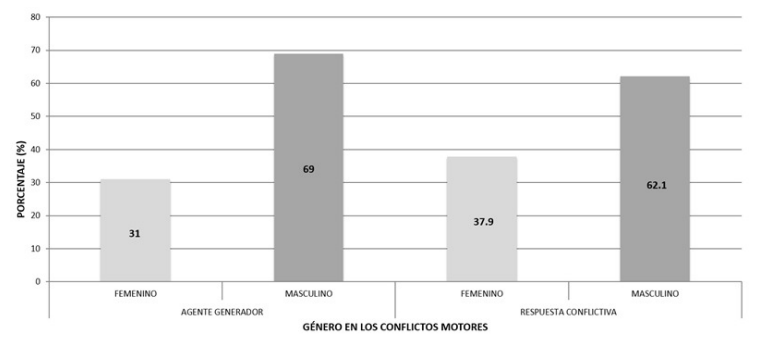

El indice de conflictividad de los conflictos motores de cooperación y de género de sus protagonistas

Atendiendo a la intensidad de los conflictos, la aplicación del índice de conflictividad (ICF) confirmó el predominio (37.3\%) de los conflictos de alta intensidad (ICF alto), mientras que los conflictos de baja intensidad (ICF bajo) estuvieron presentes en un $33.9 \%$ y los conflictos de intensidad media (ICF medio) correspondieron al $28.8 \%$ de los casos.

Las relaciones entre el ICF y el agente generador, la respuesta conflictiva y el género de los protagonistas en el conflicto motor, se analizaron mediante la prueba estadística chi- 
cuadrado de Pearson. A posteriori, en aquellas relaciones estadísticamente significativas, se estudiaron los residuos corregidos con el objetivo de profundizar en las relaciones entre variables.

Cuando el conflicto fue originado por conductas motrices perversas los conflictos alcanzaron valores de ICF altos, mientras que cuando fueron originados por conductas verbales de pacto y conductas motrices desajustadas el nivel de intensidad del conflicto fue bajo $\left(\chi^{2}(4\right.$, $n=319)=179.38, p<0.001)($ Tabla 3$)$.

\section{Tabla 3}

Tabla de contingencia entre Índice de conflictividad y agente generador

\begin{tabular}{llrrr}
\hline \multirow{2}{*}{ Agente generador } & \multicolumn{3}{c}{ Índice de conflictividad } \\
\cline { 2 - 5 } & & Bajo & \multicolumn{1}{c}{ Medio } & \multicolumn{1}{c}{ Alto } \\
\hline \multirow{2}{*}{ Conducta verbal de pacto } & Recuento & 42 & 8 & 0 \\
& Residuos corregidos & $8.2^{*}$ & $-2.2^{*}$ & $-5.9^{*}$ \\
Conducta motriz desajustada & Recuento & 66 & 18 & 23 \\
& Residuos corregidos & $7.5^{*}$ & $-3.4^{*}$ & $-4.1^{*}$ \\
Conducta motriz perversa & Recuento & 0 & 66 & 96 \\
& Residuos corregidos & $-13^{*}$ & $4.8^{*}$ & $8.2^{*}$ \\
\hline
\end{tabular}

* Relaciones estadísticamente significativas.

Al considerar el tipo de reacción, el análisis estadístico ratificó $\left(\chi^{2}(4, n=319)=248.76, p\right.$ $<0.001)$ que cuando las respuestas conflictivas fueron a través de agresiones verbales se originaron más CM de ICF Bajo y menos CM de ICF Alto, mientras que cuando fue por medio de agresiones mixtas emergieron más CM de ICF Alto y menos CM de ICF Bajo (Tabla 4).

\section{Tabla 4}

Tabla de contingencia entre índice de conflictividad y respuesta conflictiva

\begin{tabular}{llrrr}
\hline \multirow{2}{*}{ Respuesta conflictiva } & \multicolumn{3}{c}{ Índice de conflictividad } \\
\cline { 2 - 5 } & & Bajo & Medio & \multicolumn{1}{c}{ Alto } \\
\hline \multirow{2}{*}{ Agresión verbal } & Recuento & 104 & 66 & 0 \\
& Residuos corregidos & $11^{*}$ & $4.2^{*}$ & $-14.7^{*}$ \\
\multirow{2}{*}{ Agresión física } & Recuento & 4 & 18 & 29 \\
& Residuos corregidos & $-4.3^{*}$ & 1.1 & $3.2^{*}$ \\
\multirow{2}{*}{ Agresión mixta } & Recuento & 0 & 8 & 90 \\
& Residuos corregidos & $-8.5^{*}$ & $-5.4^{*}$ & $13.4^{*}$ \\
\hline
\end{tabular}

* Relaciones estadísticamente significativas.

Asimismo, no se observaron diferencias significativas en el comportamiento entre ambos sexos en los CM de Cooperación $\left(\chi^{2}(2, n=\right.$ $319)=5.46, p=0.06)$. Sin embargo, aparecía una tendencia a que los conflictos femeninos fuesen mayoritariamente de baja intensidad (ICF Bajo), mientras que cuando los protagonistas eran chicos esos niveles tendían a ser más altos (ICF Alto). En el caso de intervenir chicos y chicas aparecía una tendencia a que el nivel de conflictividad fuese de intensidad media (ICF Medio) (Figura 3).

\section{Figura 3}

El indice de conflictividad y el género en los conflictos cooperativos

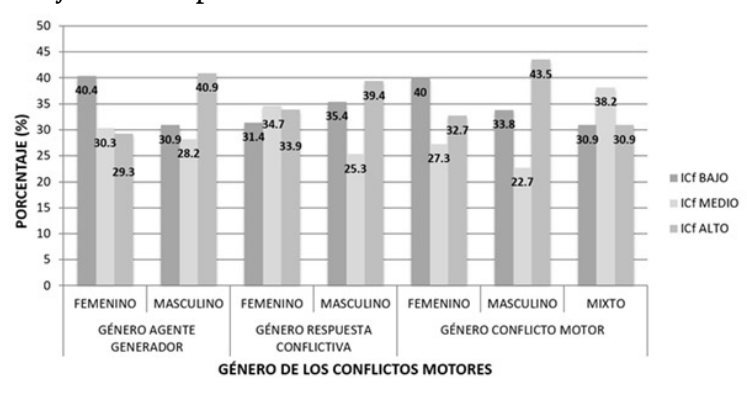

\section{Discusión}

Este estudio examinó las características de los conflictos motores que suscitaron los juegos cooperativos en el alumnado de Educación Primaria a partir de las características en su origen, reacción y en su nivel de intensidad. Los resultados confirman que el grupo estudiado es altamente conflictivo (originó conflictos en más de la mitad de los juegos). Un primer problema detectado en este grupo es la dificultad que presentan para aceptar las reglas. La lógica interna de los juegos cooperativos solicita un pacto grupal un respeto a las reglas que establecen unos mismos derechos y prohibiciones para todos los participantes (Parlebas, 2001). Esa dificultad para saber vivir en sociedad es la que origina la mayor parte de los conflictos, ya que un gran número de ellos aparecen cuando los alumnos infringen las reglas del juego (conductas motrices perversas), lo que, en el mejor de los casos, origina agresiones verbales.

La aplicación del índice de conflictividad constata el predominio de conflictos de alta intensidad en este grupo. A pesar de que se trata de juegos cooperativos, en los que las relaciones deberían ser positivas los alumnos generan tensiones en el diálogo corporal interpersonal. La lógica interna de los juegos cooperativos se 
caracteriza porque dos o más personas asumen el reto de compartir un objetivo común; para ello es necesario negociar, pactar y ponerse de acuerdo al relacionarse motrizmente (Sáez de Ocáriz \& Lavega, 2013). Sin embargo, la falta de competencias sociales de este grupo de alumnos lo lleva a generar conflictos, de ahí, el gran desafío para el colectivo de educadores de favorecer competencias tan importantes y necesarias como saber trabajar en equipo, aprender a mantener relaciones interpersonales positivas y saber tratar con respeto a las demás personas (Hromek \& Roffey, 2009; Johnson \& Johnson, 1999; Lederach, 1995; León et al., 2012).

El estudio también muestra que la mayoría de los conflictos son originados por chicos y corresponden a niveles de intensidad elevados. En cambio, las chicas actúan mediante agresiones verbales y sus conflictos tienen un nivel de intensidad medio o bajo. Estos hallazgos coinciden con otros estudios que consideran las diferencias en las relaciones interpersonales entre ambos géneros (Díaz-Aguado, 2005; DíazAguado \& Martín-Seoane, 2011; Inglés et al., 2008; Österman et al., 1998; Toldos, 2005) y la predisposición cultural desigual de los chicos y chicas para participar en juegos cooperativos (Etxebeste, 2012; Lavega, 2009; Lavega et al., 2014).

Esta investigación aplicada al contexto educativo confirma la utilidad del planteamiento seguido para caracterizar los conflictos motores. La identificación de las partes del conflicto y las características que tienen en su origen (acción) y en su respuesta (reacción) dan pistas al profesor de educación física para afrontar la transformación pedagógica de esos conflictos. Además, permiten comprender mejor la naturaleza de los conflictos interpersonales cuando se emplea la familia de juegos motores cooperativos (Sáez de Ocáriz \& Lavega, 2013).

Paralelamente, el uso del índice de conflictividad (ICF) es también un recurso pedagógico muy útil para el docente de educación física. Este indicador permite profundizar en la caracterización de los conflictos y ayuda a diagnosticar su intensidad. En este caso, se observa el predominio de conflictos de alta intensidad, lo que constata que la educación física debería tener como prioritaria la función de enseñar a los alumnos a convivir.

Estos dos criterios (identificación de las partes del conflicto y el uso del índice de conflictividad) suponen un avance para dar respuesta a las directrices de las administraciones educativas, cuando se indica que es necesario favorecer la formación del profesorado y facilitar recursos para la gestión constructiva de los conflictos. Se trata de una vía que potencia la optimización de la convivencia escolar (Ararteko, 2006; Martín et al., 2003; Penalva et al., 2014; Pérez-Gallardo et al., 2013; Sáez de Ocáriz et al., 2013).

Los resultados observados en el grupo de estudiantes investigado, plantean el desafío de transformar positivamente los conflictos, considerando que son una oportunidad para provocar cambios en las relaciones interpersonales del alumnado (Álvarez-García et al., 2010; Badia, 2009; Penalva et al., 2014). Para ello, atendiendo a criterios didácticos y pedagógicos coherentes, existen dos vías principales de actuación: intervenir sobre el juego o sobre el alumnado que protagoniza el conflicto (Sáez de Ocáriz \& Lavega, 2013).

La intervención sobre el juego comporta plantearse la modificación de las reglas o condiciones de práctica. Así, puede ser de gran utilidad plantearse el cambio del número de componentes de cada equipo; la ampliación o reducción del espacio de juego; el aumento o dismunución de la duración del juego; remplazar la manera de emplear un objeto (por ejemplo manera de pasar el balón) o el número de objetos que emplea cada grupo (por ejemplo reducir el número de balones). Estos cambios en las reglas pueden favorecer las condiciones de práctica y ayudar a los alumnos a conseguir ese desafío cooperativo. En caso que esta acción no dé resultado, se puede sustituir el juego (Johnson \& Johnson, 1999; Sáez de Ocáriz, 2011).

Paralelamente, la actuación sobre los propios protagonistas debería estar asociada a dejar que los alumnos implicados en el conflicto dispongan del tiempo necesario para gestionar esa situación conflictiva. Será interesante tratar de pasar de un modelo de ganar-vencer a uno de ganar- 
ganar (Lederach, 1995). En el primer caso, ambas partes toman el conflicto como un duelo interpersonal en el que hay que ganar a toda costa. En este segundo enfoque, las personas pueden entender que es posible actuar de otro modo, tanto en el origen como en la reacción ante un conflicto. A partir de esa comprensión, se podrá solicitar al alumnado un compromiso para futuras intervenciones (Johnson \& Johnson, 1999; Sáez de Ocáriz, 2011).

Convivir implica compartir con otras personas, con base en unos códigos sociales concretos dentro de un contexto determinado, experiencias $\mathrm{y}$ relaciones personales que traen consigo situaciones conflictivas inseparables de la convivencia democrática. Si no se valoran los conflictos emergentes como oportunidades en el proceso de enseñanza-aprendizaje, será complicado que desde los sistemas educativos se promuevan acciones a favor de su educación, como recurso para la mejora de la convivencia en los centros escolares.

En consecuencia, con el objetivo de colaborar en dicha orientación, los resultados mostrados en el presente artículo son una muestra de cómo se puede utilizar el índice de conflictividad como herramienta en la educación de los conflictos que emergen durante las sesiones de educación física, ofreciendo recursos al docente del área para abordar el tratamiento constructivo de las situaciones conflictivas, en pro de la mejora del clima de los centros educativos.

$\mathrm{Si}$ bien es cierto que la propuesta no es generalizable a todos los grupos de escolares, la experiencia descrita invita a los autores a continuar trabajando en este sentido, complementando dicha visión con el diálogo de frontera entre la educación, la psicología, la antropología, la sociología y la filosofía, para constatar la aplicabilidad de la noción de conflicto y del índice de conflictividad en otras poblaciones, así como para ofrecer al alumnado diferentes experiencias pedagógicas en las que se ponga de manifiesto la importancia y el desarrollo de competencias en la transformación de conflictos, como base para la optimización de la convivencia escolar. En este sentido, el abordaje de la educación de los conflictos en y desde la Educación Física tiene mucho que aportar.

\section{Agradecimientos}

Los autores desean agradecer al Instituto Nacional de Educación Física de Cataluña (INEFC), en sus centros adscritos a las Universidades de Lleida y de Barcelona, por el apoyo institucional y la financiación recibida para la realización de este estudio.

\section{Referencias}

Álvarez-García, D., Rodríguez, C., GonzálezCastro, P., Nun\#ez, J., \& Álvarez, L. (2010). La formacio\#n de los futuros docentes frente a la violencia escolar. Revista de Psicodidáctica, 15(1), 35-56. Recuperado de http://www.ehu.eus/ojs/ind ex.php/psicodidactica/article/view/733/608

Ararteko, Defensoría del Pueblo u Ombudsman del País Vasco. (2006). Convivencia y conflictos en los centros educativos (Informe). Vitoria-Gasteiz, País Vasco: Autor.

Ascorra, P., Bilbao, M., Gómez, G., \& Morales, M. (2016). Relación entre segregación y convivencia escolar en escuelas chilenas con altos porcentajes PISA 2009. Universitas Psychologica, 15(1), 15-27. https://doi.org/10.11144/Javeriana.u psy15-1.rsce

Badia, J. (2009). El projecte de convivència del Departament d'Educació. Educar, 43, 131-135. Recuperado de http://www.redaly c.org/pdf/3421/342130832009.pdf

Calvo, A. J., González, R., \& Martorell, C. (2001). Variables relacionadas con la conducta prosocial en la infancia y adolescencia: personalidad, autoconcepto y género. Infancia y Aprendizaje: Journal for the Study of Education and Development, 24(1), 95-112. Recuperado de https://dialnet.unir ioja.es/servlet/articulo? codigo $=48653$

Ceballos, E., Correa, N., Correa, A., Rodríguez, J., Rodríguez, B., \& Vega, A. (2012). La voz del alumnado en el conflicto escolar. Revista 
de Educación, 359, 554-579. https://doi.org/ 10.4438/1988-592X-RE-2011-359-207

Ceballos, E., Rodríguez, B., Correa, A., \& Rodríguez, J. (2016). La evaluación situacional de los conflictos: construcción y análisis del cuestionario de estrategias y metas de resolución de conflictos escolares. Educación XX1, 19(2), 273-292. https://doi .org/10.5944/educXX1.13943

Del Rincón, D., Arnal, J., Latorre, A., \& Sans, A. (1995). Técnicas de investigación en ciencias sociales. Madrid: Editorial Dykinson.

Díaz-Aguado, M. J. (2005). La violencia entre iguales en la adolescencia y su prevención desde las aulas. Psicothema, 17(4), 549-558. Recuperado de http://www.psicothema.co $\mathrm{m} / \mathrm{pdf} / 3144 . \mathrm{pdf}$

Díaz-Aguado, M. J., \& Martín-Seoane, G. (2011). Convivencia y aprendizaje escolar en la adolescencia desde una perspectiva de género. Psicothema, 23(2), 252-259. Recuperado de http://www.psicothema.co $\mathrm{m} / \mathrm{psicothema}$.asp? $\mathrm{id}=3879$

Durán Aponte, E., \& Durán García, M. (2012). Competencias sociales y las pra\#cticas profesionales. Vivencias y demandas para la formacio\#n universitaria actual. Cultura y Educación, 24(1), 61-76. https://doi.org/10. 1174/113564012799740777

Etxebeste, J. (2012). À cloche-pied. Les jeux sportifs traditionnels et la socialisation des enfants basques. Saarbrücken, DE: Èditions Universitaires Européennes.

Garaigordobil, M. (2012). Cooperative conflictsolving during adolescence: Relationship with cognitive-behavioural and predictor variables. Infancia y Aprendizaje: Journal for the Study of Education and Development, 35(2), 151-165. https://doi.org/10.1174/02 1037012800217998

Garaigordobil, M., \& Oñederra, J. A. (2009). Acoso y violencia escolar en la comunidad autónoma del País Vasco. Psicothema, 21 (1), 83-89. Recuperado de http://www.psicothe ma.com/psicothema.asp?id=3599

Gázquez, J. J., Pérez-Fuentes, M. C., Carrión, J. J., \& Santiuste, V. (2010). Estudio y análisis de conductas violentas en Educación Secundaria en España. Universitas Psychologica, 9(2), 371-380. Recuperado de http://revistas.javeriana.ed u.co/index.php/revPsycho/article/view/304

Gil-Madrona, P., \& Martínez-López, M. (2015). Emociones autopercibidas en las clases de educación física en Primaria. Universitas Psychologica, 14(3), 923-936. https://doi.org /10.11144/Javeriana.upsy14-3.eapc

Howe, K. N., \& Moses, M. S. (1999). Ethics in educational research. Review of Research in Education, 29, 21-59. https://oi.org/10.310 2/0091732X024001021

Hromek, R. \& Roffey, S. (2009). Promoting social and emotional learning with games: "It's fun and we learn things". Simulation Gaming, 40(5), 626-644. https://doi.org/10. $1177 / 1046878109333793$

Inglés, M. J., Martínez-Monteagudo, M. C., Delgado, B., Torregrosa, M., Redondo, J., Benavides, G., \& García-López, L. (2008). Prevalencia de la conducta agresiva, conducta prosocial y ansiedad social en una muestra de adolescentes españoles: un estudio comparativo. Infancia y Aprendizaje: Journal for the Study of Education and Development, 31(4), 449-461. https://doi.or $\mathrm{g} / 10.1174 / 021037008786140968$

Jares, X. R. (2006). Conflicto y convivencia en los centros educativos de secundaria. Revista de Educación, 339, 467-491. Recuperado de http://www.revistaeducacio n.educacion.es/re339/re339_21.pdf

Johnson, D. W., \& Johnson, R. T. (1999). Cómo reducir la violencia en las escuelas. Buenos Aires: Paidós.

Koçak, R. (2008). The effects of cooperative learning on psychological and social traits among undergraduate students. Social Behavior and Personality: An International Journal, 36, 771-782. https://doi.org/10.222 4/sbp.2008.36.6.771

Lagardera, F., \& Lavega, P. (2005). La educación física como pedagogía de las conductas motrices. Tándem. Didáctica de La Educación Física, 18, 79-101. Recuperado de https://dialnet.unirioja.es/se rvlet/articulo? codigo $=1173034$ 
Lavega, P. (2009). La investigación en los juegos tradicionales y en los juegos cooperativos. En V. Navarro \& C. Trigueros (Eds.), Investigación y juego motor en España (pp. 77-116). Lleida, ES: Universitat de Lleida.

Lavega, P., Planas, A., \& Ruíz, P. (2014). Juegos cooperativos e inclusión en educación física. Revista Internacional de Medicina y Ciencias de la Actividad Física y el Deporte, 14(53), 37-51. Recuperado de http://cdeporte.rediris.es/revista/revista 53/artjuegos450.htm

Led, P., \& Quera, P. (2007). La convivència en els centres d'educacion infantil $i$ primària. Barcelona: Departament d'Educació, Servei de Difusió i Publicacions.

Lederach, J. P. (1995). Preparing for peace. Conflict transformation across cultures. Nueva York: Syracuse University Press.

León, B., Gozalo, M., \& Polo, M. A. (2012). Aprendizaje cooperativo y acoso entre iguales. Infancia y Aprendizaje: Journal for the Study of Education and Development, 35(1), 23-35. https://doi.org/10.1174/0210370127 98977494

Martín, E., Fernández, I., Andrés, S., Del Barrio, C., \& Echeita, G. (2003). La intervención para la mejora de la convivencia en los centros educativos: modelos y ámbitos. Infancia y Aprendizaje: Journal for the Study of Education and Development, 26(1), 79-95. Recuperado de http://files.acosoescolarenlasaulas-org.w ebnode.es/200000027-cde02cf1c9/Martin_ et_al._2003_pdf

Mesía, R. (2007). Contexto ético de la investigación social. Investigación Educativa, 11(19), 137-151. Recuperado de http://sisbib.unmsm.edu.pe/bibVirtualD ata/publicaciones/inv_educativa/2007_n19 /a11.pdf

Molina, F. (2005). Nuevos conflictos sociales y su presencia educativa. Análisis sociológico y reflexiones para la intervención. Cultura y Educación, 17(3), 213-223. https://doi.org/ $10.1174 / 113564005774462618$
Ortega, R., \& Del Rey, R. (2003). La violencia escolar. Estrategias de prevención. Barcelona: Editorial Graó.

Österman, D., Björkqvist, D., Lagerspezt, K., Kaukiainen, A., Landau, S. F., Frączek, A., \& Caprara, G. V. (1998). Cross-cultural evidence of female indirect aggression. Aggressive Behavior, 24, 1-8. https://doi.org/10.1002/ (SICI) 1098-2337(1998) 24:1<1::AID$\mathrm{AB} 1>3.0 . \mathrm{CO} ; 2-\mathrm{R}$

Parlebas, P. (2001). Juegos, deporte y sociedad. Léxico de praxiología motriz. Barcelona: Editorial Paidotribo.

Penalva, A., Hernández, M., \& Guerrero, C. (2014). Percepcio\#n de los expertos de la necesidad de la formacio\# $\mathrm{n}$ del profesorado en convivencia. Revista Fuentes, 15, 281-304. https://doi.org/10.12795/revistafu entes.2014.i15.13

Pérez, A., \& Poveda, P. (2008). Efectos del aprendizaje cooperativo en la adaptación escolar. Revista de Investigación Educativa, 26, 73-94. Recuperado de http://revistas.u m.es/rie/article/view/94121/90741

Pérez-Gallardo, E., Álvarez-Hernández, J., Aguilar, J. M., Fernández-Campoy, J. M., \& Salguero, D. (2013). El dialogo como instrumento para la resolucio\#n de conflictos en escolares de educacio\#n secundaria. International Journal of Developmental and Educational Psychology, 1(2), 189-194. Recuperado de http://infad.eu/RevistaINFAD/2013/n1/ volumen2/INFAD_010225_189-194.pdf

Pérez-Pérez, C. (2007). Efectos de la aplicación de un programa de educación para la convivencia sobre el clima social del aula en un curso de 2. de ESO. Revista de Educación, 343, 503-529. Recuperado http://www.revistaeducacion. mec.es/re343/re343_21.pdf

Quivy, R., \& Campenhoudt, L. V. (2000). Manual de investigación en ciencias sociales. México: Editorial Limusa.

Real Decreto 1513 de 2006 [Ministerio de Educación y Ciencia de España]. Por el que se establecen las enseñanzas mínimas de la 
Educación primaria. Diciembre 7 de 2006). BOE-A-2006-21409.

Rodríguez, G., Gil, J., \& García, E. (1996). Metodología de la investigación cualitativa. Málaga: Ediciones Aljibe.

Ruiz-Olabuénaga, J. (1999). Metodología de la investigación cualitativa (2.a ed.). Bilbao: Universidad de Deusto.

Sáez de Ocáriz, U. (2011). Conflictos y educación física a la luz de la praxiología motriz. Estudio de caso de un centro educativo de primaria (Tesis doctoral inédita). Universitat de Lleida, Lleida.

Sáez de Ocáriz, U., \& Lavega, P. (2013). Transformar conflictos en educación física en primaria a través del juego. Aplicación del índice de conflictividad. Cultura y Educación, 25(4), 549-560. https://doi.org/ 10.1080/11356405.2013.10783161

Sáez de Ocáriz, U., \& Lavega, P. (2015). Estudio de los conflictos en el juego en Educación Física en Primaria. Revista Internacional de Medicina y Ciencias de la Actividad Física y el Deporte, 15(57), 29-44. https://doi.org/10.1 5366/rimcafd2015.57.003

Sáez de Ocáriz, U., Lavega, P., \& March, J. (2013). El profesorado ante los conflictos en la Educación Física. El caso de los juegos de oposición en Primaria. Revista Electrónica Interuniversitaria de Formación Del Profesorado, 16(1), 163-176. https://doi .org/10.6018/reifop.16.1.180101

Stenhouse, L. (1987). Investigación y desarrollo del curriculum. Madrid: Morata.

Torrecilla, E., Olmos, S., \& Rodríguez, MJ. (2016). Efectos de la metodología didáctica sobre el aprendizaje de competencias para la gestión de conflictos en Educación Secundaria. Educación XX1, 19(2), 293-315. https://doi.org/10.5944/ed ucXX1.13949

Torregrosa, M. S., Inglés, C., GarcíaFernández, J. M., Valle, A., \& Núñez, J. (2012). Relaciones entre conducta agresiva y metas académicas: estudio con una muestra de estudiantes españoles en Educación Secundaria Obligatoria. Universitas Psychologica,
11(4), 1303-1315. Recuperado de http://revistas.javeriana.edu.co/index.p hp/revPsycho/article/view/1303

Toldos, M. P. (2005). Sex and age differences in self-estimated physical, verbal and indirect aggression in Spanish adolescents. Aggressive Behavior, 31 (1), 13-23. https://do i.org/10.1002/ab.20034

Torrego, J., \& Galán, A. (2008). Investigación evaluativa sobre el programa de mediación de conflictos en centros escolares. Revista de Educación, 347, 369-394. Recuperado de http://www.revistaeducacion.mec.es/re3 47/re347_17.pdf

Viguer, P., \& Solé, N. (2015). La participación de las familias en el análisis y la transformación de su realidad mediante un debate familiar sobre valores y convivencia. Universitas Psychologica, 14(1), 355-366. https://doi.org /10.11144/Javeriana.upsy.13-5.1pfa

\section{Notas}

* Artículo de investigación. Los resultados que aparecen en el presente artículo complementan los ya publicados (Sáez de Ocáriz \& Lavega, 2013, 2015), donde la metodología utilizada fue similar a la aquí descrita al formar parte de una investigación común, de la cual solo se muestran los datos que dan respuesta al objeto de estudio planteado en este documento. La investigación fue financiada por el Instituto Nacional de Educación Física de Cataluña (INEFC). 